Nuclear Physics B230 [FS10] (1984) 261-272

(C) North-Holland Publishing Company

\title{
A NEW RENORMALIZATION GROUP APPROACH TO MULTISCALE PROBLEMS
}

\author{
Martin B. EINHORN and D.R. Timothy JONES ${ }^{1}$ \\ Randall Laboratory of Physics, University of Michigan, Ann Arbor, MI 48109, USA
}

Received 13 September 1983

\begin{abstract}
A new renormalization group is presented which exploits invariance with respect to more than one scale. The method is illustrated by a simple model, and future applications to fields such as critical phenomena and supersymmetry are speculated upon.
\end{abstract}

\section{Introduction}

In principle, the ground state of a theory can be determined by locating the minimum of the effective potential $V_{\text {eff }}$ (or, at finite temperature, of the free energy) [1]. In practice, one must resort to making approximations to $V_{\text {effi }}$ Aside from lattice calculations, these are generally of a perturbative nature. Typically, a coupling constant, loop, or high-temperature expansion is employed, whose convergence depends upon the magnitude of the expansion parameter. However, it sometimes happens that, even though the expansion parameter is small, the approximation to the effective potential fails because of the occurrence of large logarithms whose arguments involve the ratio of very different scales. In these cases, the situation can frequently be improved upon by summing up such logarithms, which is most efficiently performed via the renormalization group. This leads to a renormalizationgroup-improved version of the effective potential for which the perturbative expansion remains valid so long as the so-called "running" coupling constants remain small [2]. Another, not unrelated, application concerns the so-called decoupling theorems and the derivation of effective lagrangians to describe the low-energy effects of heavy particles. Here again, potentially large logarithms are conveniently reabsorbed in low-energy effective parameters and fields by means of renormalization group constraints [3].

However, there are situations in which the standard renormalization group methods are still inadequate to deal with the large logarithms which invalidate the direct application of perturbation theory. These situations arise when the problem of interest has two or more relevant but widely disparate scales. One example is in

${ }^{1}$ Address from September 1, 1983: Department of Physics, University of Colorado, Boulder, CO 80302 . 
grand unified theories generally, but especially in spontaneously broken, supersymmetric grand unified models. ${ }^{\star}$ In such models, there is always at least one field (the scalar component of the goldstino superfield) which is undetermined classically. In previous work, it has been demonstrated how, through radiative corrections, the ground state expectation value $\langle G\rangle$ can be determined [4]. However, a straightforward application of perturbation theory is not adequate to the task, and one must apply a renormalization-group-improved approximation to $V_{\text {eff }}$ to succeed. However, in the more general case when more than one linearly independent scalar field is undetermined, even the usual renormalization group does not yield a solution.

The same thing can arise in a non-supersymmetric context in which more than one field (scalar order parameter) must be fixed via radiative corrections. Although no immediate physically relevant model springs to mind, one can invent a simple generalization of the Coleman-Weinberg massless $U_{1}$ gauge theory to illustrate the point. In sect. 2 , we review the Coleman-Weinberg situation and then consider a $\mathrm{U}_{1} \times \mathrm{U}_{1}$ generalization thereof which is illustrative and shares many features with generic, supersymmetric models. The main difficulty in such applications is that, in perturbative theory, one obtains terms of the form $\log ^{m} \phi_{1} \log ^{n} \phi_{2}$, where $\phi_{1}$ and $\phi_{2}$ are two fields whose scales are to be determined. One wishes to perform a summation in each variable separately, but the standard renormalization group equation is equivalent to a summation for a fixed ratio $\phi_{1} / \phi_{2}$. If this ratio is very large or small, the remaining large logarithms of the ratio invalidates the application of perturbation theory.

\section{Review of the "massless", $U_{1}$ gauge theory}

Coleman and Weinberg ${ }^{\star \star}[2]$ consider the case of scalar, massless electrodynamics, a relativistic generalization of the Landau-Ginsburg model for superconductivity (at the critical temperature). The renormalized lagrangian takes the form

where

$$
\mathscr{L}=\left|D_{\mu} \Phi\right|^{2}-\frac{1}{4} F_{\mu \nu}^{2}-\frac{1}{6} \lambda\left(\Phi^{\dagger} \Phi\right)^{2}+\delta \mathscr{L},
$$

$$
D_{\mu} \Phi \equiv \partial_{\mu} \Phi-i e A_{\mu} \Phi,
$$

and $\delta \mathscr{L}$ represents appropriate counterterms. Even though classically, $\langle\Phi\rangle=\mathbf{0}, \mathrm{CW}$ established that $\langle\Phi\rangle \neq 0$ as a result of radiative corrections. We will review this development in order to establish our renormalization conventions (which differ from $\mathrm{CW}$ ) and to be in a position to compare this to our more complicated, two-scale model. The effective potential obeys the renormalization group equation

$$
\left[\mu \frac{5}{\partial \mu}+\beta_{\lambda} \frac{\partial}{\partial \lambda}+\beta_{e} \frac{\partial}{\partial e}+\gamma \phi \frac{\partial}{\partial \phi}\right] V_{\text {eff }}=0,
$$

\footnotetext{
* A knowledge of supersymmetry is not required for the example to be discussed in detail in sect. 2 . ** Hereafter, this reference will be abbreviated as CW.
} 
where we have chosen the classical field $\phi$ to be real. In one-loop approximation, the coefficients are given by ${ }^{\star}$

$$
\begin{aligned}
& \beta_{\lambda}=\frac{1}{16 \pi^{2}}\left[\frac{10}{3} \lambda^{2}-12 \lambda e^{2}+36 e^{4}\right], \\
& \beta_{e}=\frac{1}{16 \pi^{2}}\left(\frac{1}{3} e^{3}\right), \quad \gamma=\frac{3 e^{2}}{16 \pi^{2}} .
\end{aligned}
$$

The one-loop approximation to the effective potential takes the form

$$
V_{\mathrm{eff}}=V_{\mathrm{cl}}+\frac{\hbar}{64 \pi^{2}}\left[\operatorname{Tr} M^{4} \ln M^{2}-A \ln \mu^{2}\right],
$$

where we have employed a finite redefinition of the coupling constant $\lambda$ in order to eliminate a term proportional to $\phi^{4}$ in order $\hbar$. As usual, $M$ denotes the tree-level mass matrices. The coefficient $A$ may be obtained by direct calculation or by means of the $\beta$ and $\gamma$ functions through the renormalization group equation:

$$
\frac{A}{32 \pi^{2}}=\left[\beta_{\lambda} \frac{\partial}{\partial \lambda}+\beta_{e} \frac{\partial}{\partial e}+\gamma \phi \frac{\partial}{\partial \phi}\right] V_{\mathrm{cl}},
$$

where the classical potential is $V_{\mathrm{cl}}=\lambda \phi^{4} / 4$ ! The result is

$$
A=\left(\frac{5}{18} \lambda^{2}+3 e^{4}\right) \phi^{4} \text {. }
$$

The tree-level masses can be easily calculated, so the trace is simply given as

$$
\operatorname{Tr} M^{4} \ln M^{2}=\left(\frac{1}{2} \lambda \phi^{2}\right)^{2} \ln \left(\frac{1}{2} \lambda \phi^{2}\right)+\left(\frac{1}{6} \lambda \phi^{2}\right)^{2} \ln \left(\frac{1}{6} \lambda \phi^{2}\right)+3\left(e^{2} \phi^{2}\right)^{2} \ln \left(e^{2} \phi^{2}\right) .
$$

Although the preceding has been derived assuming $\lambda>0$, the sign of $\lambda$ is not a scale invariant concept ${ }^{\star \star}$. However, the effective potential is not analytic at $\lambda=0$, reflecting the infrared divergences present at that point. Surprisingly enough, another finite (non-analytic) renormalization of the coupling constant $\lambda$ can be exploited to remove this singularity. Defining $\lambda^{\prime}$ by ${ }^{\star \star \star}$

$$
\frac{\lambda^{\prime}}{4 !}=\frac{\lambda}{4 !}+\frac{\hbar}{64 \pi^{2}}\left(\frac{1}{4} \lambda^{2} \ln \frac{1}{2} \lambda+\frac{1}{6} \lambda^{2} \ln \frac{1}{6} \lambda+3 e^{4} \ln e^{2}\right),
$$

then we find simply $\operatorname{Tr} M^{4} \ln M^{2}=A \ln \phi^{2}$, so that

$$
V_{\text {eff }}=\frac{\lambda^{\prime} \phi^{4}}{4 !}+\frac{\hbar}{64 \pi^{2}}\left(\frac{5}{18} \lambda^{\prime 2}+3 e^{4}\right) \phi^{4} \ln \phi^{2} / \mu^{2}
$$

Now one may continue to negative $\lambda^{\prime}$ with impunity†.

\footnotetext{
* We employ minimal subtraction and use the Landau gauge for convenience.

** Indeed, with our conventions, the minimum occurs for negative $\lambda$, see below.

$\star \star \star T h i s ~ n o n-a n a l y t i c$ renormalization leads to changes in the $\beta$ functions beyond one-loop. The simplicity of the result does not generalize to the more complicated model considered below.

† Subsequently, we will drop the prime on $\lambda^{\prime}$.
} 
To establish the non-zero minimum $\langle\phi\rangle$, one may proceed in one of two ways: (i) One may calculate perturbatively if one cleverly chooses the normalization mass $\mu \equiv\langle\phi\rangle$, so that no large logarithms are encountered for small variations about this scale. Assuming that $\lambda \sim e^{4}$, we find $\partial V_{\text {eff }} / \partial \phi=0$ provided $\lambda+9 \alpha^{2}=0\left(\alpha \equiv e^{2} / 4 \pi\right)$. One may then establish that, so long as the normalization scale is chosen within the range for which $\lambda$ and $\alpha$ are small, the running coupling constants always evolve to a regime where $\lambda \sim \alpha^{2}$, that is, $\lambda \sim \alpha^{2}$ near the minimum but not elsewhere (more typically $\lambda \sim \alpha$ ).

(ii) Alternatively, if $\mu$ is not chosen near $\langle\phi\rangle$, (so that $\lambda$ is not of order $\alpha^{2}$ ), then a direct perturbative calculation is not possible because $\lambda \ln \langle\phi\rangle / \mu \sim 1$. However, one may form the "renormalization group improved" effective potential (still using a perturbative approximation for $\beta$ and $\gamma$ ) to sum up the large logarithms and thereby establish the same result as before using the first method.

Consider now a $\mathrm{U}_{1} \times \mathrm{U}_{1}$ extension of the $\mathrm{CW}$ model, with classical potential of the form

$$
V_{\mathrm{cl}}=\frac{1}{6}\left(\lambda_{1}\left(\Phi_{1}^{\dagger} \Phi_{1}\right)^{2}\right)+\frac{1}{6}\left(\lambda_{2}\left(\Phi_{2}^{\dagger} \Phi_{2}\right)^{2}\right)+\lambda_{3}\left(\Phi_{1}^{\dagger} \Phi_{1}\right)\left(\Phi_{2}^{\dagger} \Phi_{2}\right) .
$$

Note that, for $\lambda_{3}=0, \Phi_{1}$ and $\Phi_{2}$ are uncoupled and the theory becomes a direct sum of two CW models. The two ground state expectation values $\left\langle\Phi_{1}\right\rangle$ and $\left\langle\Phi_{2}\right\rangle$ are determined by dimensional transmutation, but the ratio $\left\langle\Phi_{1}\right\rangle /\left\langle\Phi_{2}\right\rangle$ is completely arbitrary. For sufficiently small $\lambda_{3}$, we expect $\left\langle\Phi_{1}\right\rangle$ and $\left\langle\Phi_{2}\right\rangle$ to be only slightly changed, but, if their ratio is sufficiently large (or small), it is impossible to establish this by either of the methods used to solve the $\mathrm{CW}$ model: (i) If we choose to normalize at say $\mu=\left\langle\Phi_{1}\right\rangle$, then in general we will not have $\lambda_{2}$ of $O\left(e_{2}^{4}\right)$, that is, we will be outside the domain of validity of perturbation theory for $\Phi_{2}$. (ii) If one improves on $V_{\text {eff }}$ by implementing consistency with the renormalization group, then one still faces logs of the ratio $\left\langle\Phi_{1}\right\rangle /\left\langle\Phi_{2}\right\rangle$, which can also become large and invalidate the approximation.

One might proceed via decoupling to solve the problem iteratively, but it is clear that a more symmetric methodology is called for. The preceding uncoupled example $\left(\lambda_{3}=0\right)$ suggests that what is required is a treatment which takes advantage of separate rescalings of linearly independent fields.

The analogue of CW's normalization conditions for the couplings would be

$$
\lambda_{1}=\left.\frac{\partial^{4} V_{\mathrm{eff}}}{\partial \phi_{1}^{4}}\right|_{\substack{\phi_{1}=\mu_{1} \\ \phi_{2}=\mu_{2}}}, \quad \lambda_{2}=\left.\frac{\partial^{4} V_{\mathrm{eff}}}{\partial \phi_{2}^{4}}\right|_{\substack{\phi_{1}=\mu_{1} \\ \phi_{2}=\mu_{2}}}, \quad \lambda_{3}=\left.\frac{\partial^{2} V_{\mathrm{eff}}}{\partial \phi_{1}^{2} \partial \phi_{2}^{2}}\right|_{\substack{\phi_{1}=\mu_{1} \\ \phi_{2}=\mu_{2}}},
$$

and similarly for $e_{1}$ and $e_{2}$. Customarily, one chooses the same normalization scale for both fields, $\mu_{1}=\mu_{2}$, but this is not necessary and, for our purposes, undesirable. One may then develop new renormalization group equations which express the

* As before, we choose phases so that the classical fields $\phi_{1}$ and $\phi_{2}$ are both real. 
invariance of the theory under independent variations of $\mu_{1}$ and $\mu_{2}$. The resulting normalization group equations take the general form

$$
\begin{aligned}
& {\left[\mu_{1} \frac{\partial}{\partial \mu_{1}}+\beta_{\lambda_{\rho}}^{(1)} \frac{\partial}{\partial \lambda_{\rho}}+\gamma_{i}^{(1)} \phi_{i} \frac{\partial}{\partial \phi_{i}}\right] V_{\mathrm{eff}}=0,} \\
& {\left[\mu_{2} \frac{\partial}{\partial \mu_{2}}+\beta_{\lambda_{\rho}}^{(2)} \frac{\partial}{\partial \lambda_{\rho}}+\gamma_{i}^{(2)} \phi_{i} \frac{\partial}{\partial \phi_{i}}\right] V_{\mathrm{eff}}=0,}
\end{aligned}
$$

where, for brevity, we define $\lambda_{\rho} \equiv\left(\lambda_{1}, \lambda_{2}, \lambda_{3}, e_{1}, e_{2}\right)$. In general, the $\beta$ functions and anomalous dimensions depend on the ratio $\mu_{1} / \mu_{2}$ as well as other dimensionless variables or ratios. In the standard development, it is advantageous to pursue a "mass-independent" approach based on dimensional regularization and minimal subtraction, yielding $\beta$ 's and $\gamma$ 's which depend only on the dimensionless coupling constants. In our new method, this technique preserves their independence of the ratio $\mu_{1} / \mu_{2}$ only in one-loop-approximation but, in higher order, such an explicit dependence appears to be unavoidable. However, the mass independence at oneloop is of considerable technical advantage and generally permits a solution of the problem closely analogous to the one-scale formalism. This is the prescription we will follow henceforth.

We have developed the general solution of such systems of equations, but we will spare the reader this rather cumbersome formalism and develop a simpler solution to the problem which is a generalization of the perturbative CW method (1) described above. We present this in sect. 3 and then, in the subsequent section, we perform a renormalization group analysis of the coupling constant flows to ascertain whether the assumptions made are general.

\section{Multiscale solution of the $U_{1} \times U_{1}$ model}

Our goal in this section is to present the solution to the $U_{1} \times U_{1}$, coupled $\mathrm{CW}$ model defined by eq. (10).

To this end we introduce three different renormalization masses for the three couplings $\lambda_{1}, \lambda_{2}, \lambda_{3}$ :

$$
\lambda_{i}^{\mathrm{B}}=\lambda_{i} \mu_{i}^{\varepsilon} Z_{\lambda_{i}}, \quad \text { (not summed over } i \text { ) }
$$

where $\lambda_{i}^{\mathrm{B}}$ are the bare coupling constants and $\varepsilon \equiv 4-n$. One could in principle introduce additional normalization masses for the gauge couplings $e_{1}$ and $e_{2}$ but it is equally convenient for our purposes to take

$$
e_{i}^{\mathrm{B}}=e_{i} \mu_{i}^{\varepsilon / 2} Z_{e_{i}} \quad \text { (not summed) }
$$

Similarly, wave-function renormalizations may be associated with additional scales, but we simply choose to employ minimal subtraction so that the renormalization mass dependence is to be inferred from the coupling constant dependence. This 
procedure enables one to determine unambiguously the "partial" $\beta$ and $\gamma$ functions which appear in the renormalization group equations. In the present case, there are three such equations

$$
\left[\mu_{\alpha} \frac{\partial}{\partial \mu_{\alpha}}+\beta_{\lambda_{\rho}}^{(\alpha)} \frac{\partial}{\partial \lambda_{\rho}}+\gamma_{i}^{(\alpha)} \phi_{i} \frac{\partial}{\partial \phi_{i}}\right] V_{\mathrm{cff}}=0,
$$

(not summed on $\alpha=1,2,3$ ). In one loop approximation, the non-zero coefficients $\operatorname{are}^{\star}$

$$
\begin{array}{ll}
\beta_{\lambda_{1}}^{(1)}=\frac{1}{16 \pi^{2}}\left[\frac{10}{3} \lambda_{1}^{2}-12 \lambda_{1} e_{1}^{2}+36 e_{1}^{4}-6 \lambda_{3}^{2}\right], & \beta_{\lambda_{3}}^{(1)}=\frac{\lambda_{3}}{16 \pi^{2}}\left[\frac{4}{3} \lambda_{1}-6 e_{1}^{2}\right], \\
\beta_{\lambda_{2}}^{(2)}=\frac{1}{16 \pi^{2}}\left[\frac{10}{3} \lambda_{2}^{2}-12 \lambda_{2} e_{2}^{2}+36 e_{2}^{4}-6 \lambda_{3}^{2}\right], & \beta_{\lambda_{3}}^{(2)}=\frac{\lambda_{3}}{16 \pi^{2}}\left[{ }_{3}^{4} \lambda_{2}-6 e_{2}^{2}\right], \\
\beta_{e_{1}}^{(1)}=\frac{1}{16 \pi^{2}}\left(\frac{1}{3} e_{1}^{3}\right), \quad \beta_{e_{2}}^{(2)}=\frac{1}{16 \pi^{2}}\left(\frac{1}{3} e_{2}^{3}\right), & \\
\beta_{\lambda_{1}}^{(3)}=\beta_{\lambda_{2}}^{(3)}=\frac{12 \lambda_{3}^{2}}{16 \pi^{2}}, \quad \beta_{\lambda_{3}}^{(3)}=\frac{4 \lambda_{3}^{2}}{16 \pi^{2}}, \\
\gamma_{1}^{(1)}=\frac{3 e_{1}^{2}}{16 \pi^{2}}, \quad \gamma_{2}^{(2)}=\frac{3 e_{2}^{2}}{16 \pi^{2}} .
\end{array}
$$

All others are zero, in particular, note for future reference that $\beta_{\lambda_{2}}^{(1)}=\beta_{\lambda_{1}}^{(2)}=0$. The effective potential may also be calculated unambiguously. In one-loop-approximation, it takes the form

$$
V_{\mathrm{P}}=V_{\mathrm{cl}}+\frac{\hbar}{64 \pi^{2}}\left[\operatorname{Tr} M^{4} \ln M^{2}-A_{\alpha} \ln \mu_{\alpha}^{2}\right],
$$

where the coefficients $A_{\alpha}$ are polynomials in the couplings and fields. They may be determined from the renormalization group to be

$$
\frac{1}{32 \pi^{2}} A_{\alpha}=\left[\beta_{\rho}^{(\alpha)} \frac{\partial}{\partial \lambda_{\rho}}+\gamma_{i}^{(\alpha)} \phi_{i} \frac{\partial}{\partial \phi_{i}}\right] V_{\mathrm{cl}} \text {. }
$$

Using the previous formulae (16), we find

$$
\begin{aligned}
& A_{1}=\left(\frac{5}{18} \lambda_{1}^{2}+3 e_{1}^{4}-\frac{1}{2} \lambda_{3}^{2}\right) \phi_{1}^{4}+\frac{2}{3} \lambda_{1} \lambda_{3} \phi_{1}^{2} \phi_{2}^{2}, \\
& A_{2}=\left(\frac{5}{18} \lambda_{2}^{2}+3 e_{2}^{4}-\frac{1}{2} \lambda_{3}^{2}\right) \phi_{2}^{4}+\frac{2}{3} \lambda_{2} \lambda_{3} \phi_{1}^{2} \phi_{2}^{2}, \\
& A_{3}=\lambda_{3}^{2}\left(\phi_{1}^{2}+\phi_{2}^{2}\right)^{2} .
\end{aligned}
$$

* We have calculated these in the Landau gauge using a minimal subtraction renormalization prescription. 
The exact computation of $\operatorname{Tr} M^{4} \ln M^{2}$ is analytically rather complicated. We find

$$
\begin{aligned}
\operatorname{Tr} M^{4} \ln M^{2}= & M_{+}^{4} \ln M_{+}^{2}+M_{-}^{2} \ln M_{-}^{2}+M_{1}^{4} \ln M_{1}^{2}+M_{2}^{4} \ln M_{2}^{2} \\
& +3\left(e_{1}^{2} \phi_{1}^{2}\right)^{2} \ln \left(e_{1}^{2} \phi_{1}^{2}\right)+3\left(e_{2}^{2} \phi_{2}^{2}\right)^{2} \ln \left(e_{2}^{2} \phi_{2}^{2}\right),
\end{aligned}
$$

where

$$
\begin{aligned}
& M_{1}^{2} \equiv \frac{1}{6} \lambda_{1} \phi_{1}^{2}+\frac{1}{2} \lambda_{3} \phi_{2}^{2}, \\
& M_{2}^{2} \equiv \frac{1}{6} \lambda_{2} \phi_{2}^{2}+\frac{1}{2} \lambda_{3} \phi_{1}^{2} .
\end{aligned}
$$

$M_{ \pm}^{2}$ are the two eigenvalues of the mass ${ }^{2}$ matrix

$$
M^{2}=\left(\begin{array}{cc}
\frac{1}{2}\left(\lambda_{1} \phi_{1}^{2}+\lambda_{3} \phi_{2}^{2}\right) & \lambda_{3} \phi_{1} \phi_{2} \\
\lambda_{3} \phi_{1} \phi_{2} & \frac{1}{2}\left(\lambda_{2} \phi_{2}^{2}+\lambda_{3} \phi_{2}^{2}\right)
\end{array}\right) .
$$

Just as in the CW model, the signs of $\lambda_{1}$ and $\lambda_{2}$ are not scale invariant and, as we shall see, the minimum again occurs for negative values of these couplings. It turns out that $M_{1}^{2}, M_{2}^{2}, M_{+}^{2}$, and $M_{-}^{2}$ are all negative at the minimum, so the effective potential is complex for $\phi_{1}$ and $\phi_{2}$ near the minimum. One is tempted to define these coupling constants as in the $\mathrm{CW}$ model to avoid this problem, but the natural replacement $\left(M_{+}^{2} \rightarrow M_{+}^{2} / \lambda_{1}, M_{1}^{2} \rightarrow M_{1}^{2} / \lambda_{1}, M_{-}^{2} \rightarrow M_{-}^{2} / \lambda_{2}, M_{2}^{2} \rightarrow M_{2}^{2} / \lambda_{2}\right)$ does not correspond to a possible finite change in counterterms because $M_{+}^{2}$ and $M_{-}^{2}$ are not fourth-order polynomials in the fields. However, one can rescale all scalar mass by the same factor $\left(M^{2} \rightarrow M^{2} / \lambda_{1}\right.$, say) since $M_{+}^{2}+M_{-}^{2}$ is a fourth-order polynomial. As we shall soon see, this technical nicety can be ignored to one-loop approximation, but it must be borne in mind if one wished to carry this calculation to higher order.

The preceding formalism is not much more complicated than the standard treatment, except that there are three normalizations scales $\mu_{i}$ and three sets of $\beta$ functions and anomalous dimensions. However, we shall now demonstrate that it is precisely this freedom which allows a perturbative solution of the model regardless of how large or small the ratio $\left\langle\phi_{1}\right\rangle /\left\langle\phi_{2}\right\rangle$ might be.

Let us suppose that $\lambda_{1} \sim e_{1}^{4}, \lambda_{2} \sim e_{2}^{4}$, and $\lambda_{3}$ also sufficiently small ${ }^{\star}$ so that all terms other than those coming from the vector bosons may be ignored. (These suppositions are to be regarded as constraints on the choice of scales $\mu_{1}, \mu_{2}$, and $\mu_{3}$.)

Then

$$
\mathrm{V}_{\mathrm{eff}} \approx V_{\mathrm{cl}}+\frac{3 \hbar}{64 \pi^{2}}\left[\left(e_{1}^{2} \phi_{1}^{2}\right)^{2} \ln \frac{e_{1}^{2} \phi_{1}^{2}}{\mu_{1}^{2}}+\left(e_{2}^{2} \phi_{2}^{2}\right)^{2} \ln \frac{e_{2}^{2} \phi_{2}^{2}}{\mu_{2}^{2}}\right] .
$$

Supposing $\left\langle\phi_{1}\right\rangle$ and $\left\langle\phi_{2}\right\rangle$ are both non-zero, it is especially convenient to choose $\mu_{1}=e_{1}\left\langle\phi_{1}\right\rangle$ and $\mu_{2}=e_{2}\left\langle\phi_{2}\right\rangle$. Then extrema are determined from the vanishing of

$$
\begin{gathered}
\frac{\partial V}{\partial \phi_{1}}=\phi_{1}\left(\frac{1}{6} \lambda_{1} \phi_{1}^{2}+\frac{1}{2} \lambda_{3} \phi_{2}^{2}+\frac{3}{2} \hbar \alpha_{1}^{2} \phi_{1}^{2}\right), \\
\frac{\partial V}{\partial \phi_{2}}=\phi_{2}\left(\frac{1}{6} \lambda_{2} \phi_{2}^{2}+\frac{1}{2} \lambda_{3} \phi_{1}^{2}+\frac{3}{2} \hbar \alpha_{2}^{2} \phi_{2}^{2}\right) .
\end{gathered}
$$

* As we shall see shortly, this requirement is $\lambda_{3} \leqslant e_{1}^{2} e_{2}^{2}$. 
Hence we find

$$
\frac{\left\langle\phi_{1}^{2}\right\rangle}{\left\langle\phi_{2}^{2}\right\rangle}=\frac{-3 \lambda_{3}}{\lambda_{1}+9 \alpha_{1}^{2}}=\frac{\lambda_{2}+9 \alpha_{2}^{2}}{-3 \lambda_{3}} .
$$

Note that this implies that $\left|\lambda_{3}\right| \leqslant \alpha_{1} \alpha_{2}$, as asserted earlier. From the matrix of second derivatives, it is easy to establish that this is indeed a local minimum. As in the CW model, these relations illustrate dimensional transmutation. The original theory has five dimensionless parameters $\left(\lambda_{1}, \lambda_{2}, \lambda_{3}, e_{1}, e_{2}\right)$. We may exchange two of these for the two scales $\left\langle\phi_{1}\right\rangle$ and $\left\langle\phi_{2}\right\rangle$ and three dimensionless couplings which we may choose to be $\lambda_{1}, \lambda_{2}, \lambda_{3}$ or $\alpha_{1}, \alpha_{2}, \lambda_{3}$ with the relations among them given by eq. (23). The specification of the quantum theory also requires that we give the value of $\mu_{3}$, but the renormalization group invariance assures us that physical predictions are independent of the choice we make for this scale. We have been cavalier about dropping all the other terms in $\operatorname{Tr} M^{4} \ln M^{2}$, but one must now check the selfconsistency of this approximation. The only cause for concern would be the occurrence of large logarithms such as $\log \left(\left\langle\phi_{1}\right\rangle /\left\langle\phi_{2}\right\rangle\right)$ or $\log \left(\left\langle\phi_{2}\right\rangle / \mu_{3}\right)$. Let us suppose in fact that $\left\langle\phi_{1}\right\rangle \gg\left\langle\phi_{2}\right\rangle$, so that the theory involves very different physical scales. Since

$$
\frac{-3 \lambda_{3}\left\langle\phi_{1}\right\rangle^{2}}{\left\langle\phi_{2}\right\rangle^{2}}=\lambda_{2}+9 \alpha_{2}^{2}
$$

we clearly must have $\left|\lambda_{3}\right| \leqslant\left[\left\langle\phi_{2}\right\rangle^{2} /\left\langle\phi_{1}\right\rangle^{2}\right] \alpha_{2}^{2}$, that is, very different scales requires a very small value of $\left|\lambda_{3}\right|$. Then one can easily see that $M_{+}^{2} \sim M_{1}^{2} \sim \lambda_{1} \phi_{1}^{2}$ and $M_{-}^{2} \sim$ $M_{2}^{2} \sim \lambda_{2} \phi_{2}^{2}$, so we find the terms in question to be

$$
\frac{\hbar}{128 \pi^{2}} \lambda_{3}^{2}\left(\phi_{1}^{4} \ln \left(\phi_{1}^{2} / \mu_{3}^{2}\right)+\phi_{2}^{4} \ln \left(\phi_{2}^{2} / \mu_{3}^{2}\right)\right.
$$

The validity of the perturbative calculation depends on showing that this is a small correction to the classical potential on both scales $\left\langle\phi_{1}\right\rangle$ and $\left\langle\phi_{2}\right\rangle$,

$$
\begin{aligned}
& \frac{1}{8} \hbar\left(\frac{\lambda_{3}}{4 \pi}\right)^{2} \ln \left(\left\langle\phi_{1}^{2}\right\rangle / \mu_{3}^{2}\right) \ll \lambda_{1}, \\
& \frac{1}{8} \hbar\left(\frac{\lambda_{3}}{4 \pi}\right)^{2} \ln \left(\left\langle\phi_{2}^{2}\right\rangle / \mu_{3}^{2}\right) \ll \lambda_{2} .
\end{aligned}
$$

Recall that these couplings depend on the normalization scales $\mu_{1}=e_{1}\left\langle\phi_{1}\right\rangle, \mu_{2}=$ $e_{2}\left\langle\phi_{2}\right\rangle$, and $\mu_{3}$. So the validity of this relation depends on whether, for some choice of $\mu_{3}$, the coupling constants obey all the relations hypothesized. To this end, we must understand the behavior of the remaining coupling constants with variations of scale. In sect. 4 , we shall show that the preceding conditions, eq. (26), can always be satisfied as well as $\lambda_{1} \sim \alpha_{1}^{2}, \lambda_{2} \sim \alpha_{2}^{2}$ so that, the preceding treatment gives the unique ground state. 


\section{Characteristic trajectories for the coupling constants}

In obtaining the perturbative solution in sect. 3, we have hypothesized that $\lambda_{1} \sim \alpha_{1}^{2}$, $\lambda_{2} \sim \alpha_{2}^{2}$, and $\lambda_{3}$ sufficiently small. The purpose of the following discussion is to show that starting anywhere in the perturbative domain, by variations of scale, we can arrange for these assumptions to be fulfilled. Just as in the CW model, we shall establish that this is indeed the case and, therefore, a renormalization group improved analysis of the effective potential would yield precisely the results obtained in the previous sections. This shows that this is the unique ground state, regardless of the magnitudes of coupling constants initially! The equations to be analyzed are

$$
\begin{aligned}
& \mu_{i} \frac{\partial}{\partial \mu_{i}} \lambda_{j}=\beta_{\lambda_{j}}^{(i)}, \quad \text { not summed on } i=1,2,3 ; j=1,2,3, \\
& \mu_{i} \frac{\partial}{\partial \mu_{i}} e_{k}=\beta_{e_{k}}^{(i)}, \quad i=1,2,3 ; k=1,2,
\end{aligned}
$$

where the non-zero $\beta$-functions are listed in eq. (16). One might at first think that having to analyze three different rescaling equations would be far more complicated than a single one, but in fact the opposite is true. The ability to vary some scales and not others allows a simple, stepwise solution of the problem. To see this, suppose we are given initial values for all five couplings $\lambda_{1}^{0}, \lambda_{2}^{0}, \lambda_{3}^{0}, e_{1}^{0}, e_{2}^{0}$, at scales $\mu_{1}^{0}, \mu_{2}^{0}$, $\mu_{3}^{0}$. What are the values at other scales?

Note first that $\lambda_{3}=0$ is a fixed point of the equations (uncoupled CW models), so the sign of $\lambda_{3}$ is renormalization group invariant. Let us consider the variations of $\lambda_{3}$ with changes of $\mu_{3}$ :

$$
\mu_{3} \frac{\partial}{\partial \mu_{3}} \lambda_{3}=\frac{\lambda_{3}^{2}}{4 \pi^{2}}
$$

with general solution

$$
\frac{1}{\lambda_{3}\left(\mu_{1}, \mu_{2}, \mu_{3}\right)}=\frac{1}{\lambda_{3}\left(\mu_{1}, \mu_{2}, \mu_{3}^{0}\right)}-\frac{1}{4 \pi^{2}} \ln \frac{\mu_{3}}{\mu_{3}^{0}} .
$$

Therefore, if $\lambda_{3}\left(\mu_{1}, \mu_{2}, \mu_{3}^{0}\right)$ is positive (negative) then by choosing $\mu_{3}$ sufficiently small (large), we may make $\lambda_{3}$ arbitrarily small. Since the sign of $\lambda_{3}\left(\mu_{1}, \mu_{2}, \mu_{3}^{0}\right)$ is the same as the sign of $\lambda_{3}^{0}$, this conclusion will be independent of the change with scales $\mu_{1}$ and $\mu_{2}$. This already answers the question of the dangerously large logarithms encountered at the end of sect. 3 . Since $\lambda_{3} \rightarrow\left(\ln \mu_{3}\right)^{-1}$ asymptotically, one can always guarantee the validity of the inequalities in eq. (26) by proper choice of $\mu_{3}$. This variation in $\mu_{3}$ causes a negligible change in $\lambda_{1}$ and $\lambda_{2}$. For example, since

$$
\mu_{3} \frac{\partial}{\partial \mu_{3}} \lambda_{1}=\frac{3}{4 \pi^{2}} \lambda_{3}^{2}=3 \mu_{3} \frac{\partial}{\partial \mu_{3}} \lambda_{3},
$$


we conclude

$$
\lambda_{1}\left(\mu_{1}, \mu_{2}, \mu_{3}\right)=\lambda_{1}\left(\mu_{1}, \mu_{2}, \mu_{3}^{0}\right)+3\left(\lambda_{3}\left(\mu_{1}, \mu_{2}, \mu_{3}\right)-\lambda_{3}\left(\mu_{1}, \mu_{2}, \mu_{3}^{0}\right) .\right.
$$

Since we are choosing $\mu_{3}$ to make $\lambda_{3}$ small, $\lambda_{1}$ is changed very little.

Now we must consider the variations induced by changes in $\mu_{1}$ or $\mu_{2}$. Consider first varying $\mu_{1}$ : Since we may neglect the $\lambda_{3}$ term in $\beta_{\lambda_{1}}^{(1)}$, the variation of $\lambda_{1}$ with $\mu_{1}$ is just as in the $\mathrm{CW}$ model! So for a change in scale of $\mu_{1}$ corresponding to a small change of $e_{1}^{2}\left(\Delta e_{1}^{2} \leqslant \frac{1}{4} e_{1}^{2}\right), \lambda_{1}$ varies over the whole real axis. In particular, we may choose $\mu_{1}$ so that $\lambda_{1} \sim e_{1}^{4}$. (We know the minimum is near the scale where $\lambda_{1} \approx-9 \alpha_{1}^{2}$.) It is crucial that this variation induces a small change in $\lambda_{3}$, so we must examine

$$
\mu_{1} \frac{\partial}{\partial \mu_{1}} \lambda_{3}=\beta_{\lambda_{3}}^{(1)}=\frac{\lambda_{3}}{16 \pi^{2}}\left(\frac{4}{3} \lambda_{1}-6 e_{1}^{2}\right)
$$

From the explicit solution for $\lambda_{1}$ as a function of $e_{1}^{2}$ (see CW, eq. (5.50)), one can explicitly solve for $\lambda_{3}$. However, this is unnecessary, since one observes that the variations of $\lambda_{3}$ is little changed by small changes in $e_{1}^{2}$. Also since $\beta_{\lambda_{2}}^{(1)}=0, \lambda_{2}$ remains unchanged by variations of $\mu_{1}$. Now, without changing $\lambda_{1}$, we may vary $\mu_{2}$ to bring $\lambda_{2}$ to order $e_{2}^{4}$, still changing $\lambda_{3}$ negligibly. Thus we have satisfied all hypotheses of sect. 3 and established that the basin of attraction of the minimum found there is the entire perturbative domain, a powerful result.

\section{Higher-order corrections}

Even in a "modified minimal subtraction" scheme such as the one employed here, the $\beta$ functions develop explicit dependence on the normalization mass ratios $\left(\mu_{1} / \mu_{3}\right.$ and $\mu_{2} / \mu_{3}$ in our model) beyond one-loop order. ${ }^{\star}$ Since these ratios might be very large or very small, one can be justifiably concerned about the stability of our procedure in higher order; whether, for example, fine tunings of couplings are required to maintain a large ratio $\left\langle\phi_{1}\right\rangle /\left\langle\phi_{2}\right\rangle$. We have not made an exhaustive investigation of this question, but at least in the particular model discussed here, it seems unlikely that higher-order corrections create problems of this sort. In a minimal subtraction scheme, dependence on normalization mass ratios enter only as the logarithm of these quantities. Moreover, we know that, for $\lambda_{3}=0$, no dependence on $\log \left(\mu_{1} / \mu_{2}\right)$ occurs since this limit corresponds to two, uncoupled, minimally subtracted models with mass-independent $\beta$-functions. Therefore, the only form in which these ratios enter the $\beta$ functions in higher order will be as $\lambda_{3} \log \left(\mu_{1} / \mu_{3}\right)$ or $\lambda_{3} \log \left(\mu_{2} / \mu_{3}\right)$. This could be dangerous since $\lambda_{3} \rightarrow\left(\log \mu_{3}\right)^{-1}$ asymptotically, so such products approach a constant and, hence, might compete with lower-order contributions. In fact, an explicit calculation of the two-loop $\beta$

\footnotetext{
* We have not explored whether a subtraction scheme can be invented to remove these dependences.
} 
functions in the ungauged version of our model reveals that these logarithms enter only with coefficients proportional to $\lambda_{3}^{2} \lambda_{2}$ or $\lambda_{3}^{2} \lambda_{1}$. Just as in our discussion of $V_{\text {eff }}$, we find that $\lambda_{3}^{2} \log \mu_{3} \rightarrow\left(\log \mu_{3}\right)^{-1}$ and so is a harmless factor regardless of how large or small the ratio $\mu_{1} / \mu_{3}$ or $\mu_{2} / \mu_{3}$ is taken to be. The reason for this fortuitous circumstance is not apparent to us, but it is encouraging.

\section{Conclusions}

In this paper, we have introduced a new renormalization group method, capable of summing up several different logarithmic scale dependences. Our "partial" renormalization group equations contain more information than the standard, singlescale renormalization group equations. We have illustrated their utility in a toy model of the Coleman-Weinberg type involving two fields both of whose scales must be determined via radiative corrections. Our method renders a perturbative solution of this model possible and makes an analysis of the renormalization group flows of the coupling constants much easier than in the standard treatment.

At present, this is a method in search of more physically relevant applications. The most immediate one which springs to mind (and which provided our initial motivation) is in globally supersymmetric models having more than one field which are classically undetermined. Elsewhere, we have indicated how, depending on the pattern of symmetry breaking, such models may avoid supersymmetric decoupling [5]. The pattern of symmetry breaking must be determined by radiative corrections and may involve widely different scales. The motivation also persists in certain supergravity models where radiative corrections may still play a crucial role [6].

Another application might be to decoupling theorems of spontaneously broken gauge theories. One may, by our techniques, be able to discuss the physics on several scales simultaneously. The large logarithms which are summed up to obtain the effective low-energy lagrangian [3] might be avoided as in the example discussed in this paper.

There are a variety of applications of perturbative QCD which are in doubt because of the occurrence of large logarithms from very different regions of phase space. (These are generally associated with the conjectured suppression of certain contributions because of Sudakov form factors [7].) Perhaps a separation of the problem into two scales, with independent renormalization group equations for each, would allow new insight into the leading-log summations involved.

In general, whenever summations of logarithms involving different arguments are required, we would expect our multiscale renormalization group method to be useful.

It would be especially interesting to find a condensed matter problem in which the radiative corrections on two very different scales or involving several independent degrees of freedom are important. As yet, we are not aware of such a realistic example. 


\section{References}

[1] D. Amit, Field theory, the renormalization group, and critical phenomena, (McGraw-Hill, NY, 1978); lectures by C.G. Callan, B.W. Lee, D.J. Gross and E. Brezin in eds. R. Balian and J. Zinn-Justin, Methods in field theory, Les Houches 1975 Summer School, Session 28 (Amsterdam, North-Holland, 1976)

[2] S. Coleman and E. Weinberg, Phys. Rev. D7 (1973) 1888

[3] Y.-P. Yao in 2nd Workshop on grand unification (Ann Arbor, Boston, Birkhäuser, 1981); lectures at 1981 Hefei Summer School on particle physics, University of Michigan UM HE 82-2, May, 1981

[4] M.B. Einhorn and D.R.T. Jones, Nucl. Phys. B211 (1983) 29;

K. Yamagishi, Nucl. Phys. B216 (1983) 508

[5] M.B. Einhorn and D.R.T. Jones, Phys. Lett. 128B (1983) 174

[6] B.A. Ovrut and S. Raby, Phys. Lett. 121B (1983) 381

[7] C.T.C. Sachrajda, Introduction to perturbative quantum chromodynamics, University of Southampton SHEP 81/82-2;

A.H. Mueller, Perturbative QCD at high energies, Phys. Reports 73 (1981) 237 